The Magnetic Field and Inductance Coefficients of Circular, Cylindrical, and Helical Currents

This content has been downloaded from IOPscience. Please scroll down to see the full text. 1906 Proc. Phys. Soc. London 20476

(http://iopscience.iop.org/1478-7814/20/1/334)

View the table of contents for this issue, or go to the journal homepage for more

Download details:

IP Address: 160.36.178.25

This content was downloaded on 01/10/2015 at 00:54

Please note that terms and conditions apply. 
XXXIII. The Magnetic Field and Inductance Coefficients of Circular, Cylindrical, and Helical Currents. By Alexasder Russeli, M.A., M.T.E.E.**

1. Introduction.

\section{TABLE of CONTunts.}

2. Mathematical Formulæ.

3. Formule for the maguetic force at any point due to the curent in a circular filament in rectangular, polar, and bipolar coordinates.

4. Applicutions of the Formulæ.

i. Force in the plane of a circular current.

ii. The nutual inductance of two coaxicl circulnr currents.

iii. Approximate formula for the self-inductance of a circular current.

5. Applications to Hydrodynamics.

6. The magnetic force due to a cylindrical current sheet.

7. Formulie for the mutual inductauce between two coaxial cylindrical current sheets.

8. Formulæ for the mutual inductance between a lielical current and it conxial cylindrical current sheet.

9. Formula for the self-inductance of a belical current, when the pitch of the belix is small.

\section{Introduction.}

Sunies formule ${ }^{+}$are wstally given for the components of the magnetic force at a point in the neighbourhood of a current flowing in a circular filament. They are generally deduced from the expression for the magnetic potential at the point expressed in a series of spherical harmonics. In many cases the series converge very slowly. It seemed desirable, therefore, to attempt to find formulae which can be evaluated more readily.

By means of Laplace's formula $\ddagger$ for the magnetic force at any point due to an element of current, formulæ for the component forces can be found very easily in terms of

* Read Fubruary 8, 1007.

+ E. Mascart and J. Joubert, Leçme sur' l'Electricité et le Magnetisme, rol. ii. $\$ 566(1897)$.

† A. Russell, 'Alternating Currents,' rol, i. p, 20). This formulat is srinetimes attributed to Ampère in Inglish text-books. 
elliptic integrals, the values of which are given in Mathematical Tables*. The results found are also useful in hydrodynamics in connexion with the theory of the circular vortex filament. The formula give directly the mutual inductance betwesn two coaxial circular currents and a close approximation to the self-inductance of a thin circular current. The autbor las not found it necessary to assume Neumann's theorem in proving any formula for self or mutual inductance. An expression is found for the axial magnetic force at any point due to a cylindrical current sheet, and particular cases are noticed. It is proved that the mutual inductance between two cylindrical current sheets is the same as that between one of them and a certain helical current of the same diameter and axial length as the other sheet. This theorem can also be immediately deduced from a formula given by Viriamu Jones $\dagger$.

The exact formula for the mutual inductance between a cylindrical current sheet and a coaxial helical filament is expressed both in terms of elliptic integrals and in a series which in general converges rapidly. Viriamu Jones left the solution in the form of a definite integral. By the formulæ given in his paper $\ddagger$ this can be expressed without difficulty in terms of complete and incomplete elliptic integrals. By utilising Jones's results, Professor Coffin in the 'Bulletin of the Bureau of Standards' (p. 118, June 1906) has done this when the axial length of the two helices is the same. The author, however, starting from Laplace's formula, directly deduces the complete solution in forms adapted for easy computation. Iorenz's formula $\S$ for the self-inductance of a helical filament is a particular case of the general formula for mutual inductance given in this paper. It is shown also that a well-known formula due to Lord Rayleigh is a particular case of Lorenz's formula.

\section{Mathematical Formulce.}

It is convenient to collect together for reference the mathematical definitions and theorems in connexion with

* For instance, Dale's 'Mathematical T'ables,' p. 76.

+ Proc. Roy. Soc. p. 203 (1898).

$\ddagger$ L. centur.

\$ Wiedeman's Amulen, vii. p. 1 ro (1879). 
Elliptic Functions which we shall require in proving the theorems which follow. Most of them are well known, and they are nearly all to be found in Legendre's Traité des Fonctions Elliptiques (1825). For proofs the reader is referred to A. Cayley's 'Elementary Treatise on Elliptic Functions.'

The definitions of the complete elliptic integrals $\mathrm{E}$ and F are

$$
\mathrm{E}=\int_{0}^{\pi / 2} \Delta d \phi, \quad \text { and } \mathrm{F}=\int_{0}^{\pi / 2} \frac{d \phi}{\Delta},
$$

where $\Delta=\left(1-k^{2} \sin ^{2} \phi\right)^{1 / 2}$, and $k$ is the modulus.

It follows at once from the Integral Calculus that

$$
\begin{aligned}
& \mathrm{E}=\frac{\pi}{2}\left\{1-\left(\frac{1}{2}\right)^{2} k^{2}-\left(\frac{1.3}{2.4}\right)^{2} \frac{k^{4}}{3}-\left(\frac{1.3 .5}{2.4 .6}\right)^{2} \frac{k^{6}}{5}-\ldots\right\} \\
& \mathrm{F}=\frac{\pi}{2}\left\{1+\left(\frac{1}{2}\right)^{2} k^{2}+\left(\frac{1.3}{2.4}\right)^{2} k^{4}+\left(\frac{1.3 .5}{2.4 .6}\right)^{2} k^{6}+\ldots\right\} .
\end{aligned}
$$

Hence when $k$ is small $E$ and $F$ can be readily computed. When $k$ is nearly equal to unity (which is its maximum value) Legendre * Las shown that we can use the formulæ

$$
\begin{aligned}
\mathrm{E}=1+ & \frac{1}{2} k_{1}{ }^{2}\left(\log _{\varepsilon} \frac{4}{k_{1}}-\frac{1}{1.2}\right) \\
& +\frac{1^{2} \cdot 3}{2^{2} \cdot 4} k_{1}^{4}\left(\log _{\varepsilon} \frac{4}{k_{1}}-\frac{2}{1.2}-\frac{1}{3.4}\right)+\ldots, \\
\mathrm{F}=\log _{\varepsilon} \frac{4}{k_{1}} & +\left(\frac{1}{2}\right)^{2} k_{1}^{2}\left(\log _{\varepsilon} \frac{4}{k_{1}}-\frac{2}{1.2}\right) \\
& +\left(\frac{1.3}{2.4}\right)^{2} k_{1}^{4}\left(\log _{\epsilon} \frac{4}{k_{1}}-\frac{2}{1.2}-\frac{2}{3.4}\right)+\ldots,
\end{aligned}
$$

where $h_{1}^{2}=1-k^{2}$, and so $k_{1}$ is a small quantity. For instance, when $k=0.99, k_{1}=0.14$ approximately. For a proof of (1) and (2) see A. Cayley, 'Elliptic Functions,' 2nd edition, p. 54.

* A. le Gendre (A. M. Jegendre), "Mémoire sur les Intégrations par" arcs d'ellipse," Histoire de l'Acadénie Royale des Sciences, 1786. 
The following theorems can be easily proved by elementary Integral Calculus :-

$$
\begin{aligned}
\int_{0}^{\pi / 2} \frac{\sin ^{2} \phi}{\Delta} d \phi & =\frac{1}{k^{2}}(\mathrm{~F}-\mathrm{E}), \ldots . . . \\
\int_{0}^{\pi / 2} \frac{\cos 2 \phi}{\Delta} d \phi & =\frac{2}{k^{2}}(\mathrm{E}-\mathrm{F})+\mathrm{F}, \ldots . \\
\int_{0}^{\pi / 2} \frac{\sin ^{2} \phi \cos ^{2} \phi}{\Delta} d \phi & =\frac{2-k^{2}}{3 k^{4}} \mathrm{E}-\frac{2-2 k^{2}}{3 k^{4}} \mathrm{~F}, . \\
\int_{0}^{\pi / 2} d \phi & =\frac{\mathrm{E}}{\Delta^{3}}, . . \\
\int_{0}^{\pi / 2} \frac{\sin ^{2} \phi d \phi}{\Delta^{3}} & =\frac{\mathrm{E}}{k^{2}\left(1-k^{2}\right)}-\frac{\mathrm{F}}{k^{2}}, \ldots . . \\
\int_{0}^{\pi / 2} \sin ^{2} \phi \Delta d \phi & =\frac{2 k^{2}-1}{3 k^{2}} \mathrm{E}+\frac{1-k^{2}}{3 k^{2}} \mathrm{~F} .
\end{aligned}
$$

The following theorems for transforming the modulus of elliptic functions are of fundamental importance. By their means simple formulæ can be foundifrom which, as Legendre has shown, the elliptic functions can be calculated to any required degree of accuracy with extreme ease. The first formula * was given by Landen.

$$
\left.\begin{array}{l}
\mathrm{F}=\left(1+k^{\prime}\right) \mathrm{F}^{\prime} \ldots . . \\
\mathrm{E}=\frac{2}{1+k^{\prime}} \mathrm{E}^{\prime}-\left(1-k^{\prime}\right) \mathrm{F}^{\prime}
\end{array}\right\}, \ldots .
$$

where the modulus of $\mathrm{E}^{\prime}$ and $\mathrm{F}^{\prime}$ is $k^{\prime}$, and

$$
k^{\prime}=\left(1-\sqrt{1-k^{2}}\right) /\left(1+\sqrt{1-k^{2}}\right)
$$

and

$$
h=2 \sqrt{k^{i}} /\left(1+k^{\prime}\right) \text {. }
$$

3. Formule for the magnetic force at any point due to the current in a circular filament in rectangular, polar, and bipolar coordinates.

In fig. $1, \mathrm{BR}$ is the conducting circular filament and $\mathrm{OZ}^{\prime}$ is its axis. It is obvious from symmetry that the lines of force

* A proof is given in Todhunter's ' Integral Calculus,' Chnpter X. 
will all be in planes passing through $O Z^{\prime}$. We shall first find the component forces $\mathrm{Z}$ and $\mathrm{X}$, parallel to $\mathrm{OZ}^{\prime}$ and $O \mathrm{X}^{\prime}$ respectively, at the point $\mathrm{P}$ in the plane $\mathrm{Z}^{\prime} \mathrm{OX}$ '. Let the radius of the circular filament be $a$, let $\mathrm{PO}$ be $r$, and let the angles $\mathrm{POX}^{\prime}$ and $\mathrm{X}^{\prime} \mathrm{OR}$ be $\theta$ and $\phi$ respectively. We shall consider the force at $P$ due to the current in an element add of the filament at $\mathrm{R}$. Let RS be the tangent to the circl, at $R$, and draw PK and KL perpendicular to $O X^{\prime}$ and $R i$ respectively. Then, noticing that $\mathrm{KL}=a-r \cos \theta \cos \phi$, and

Fig. 1.

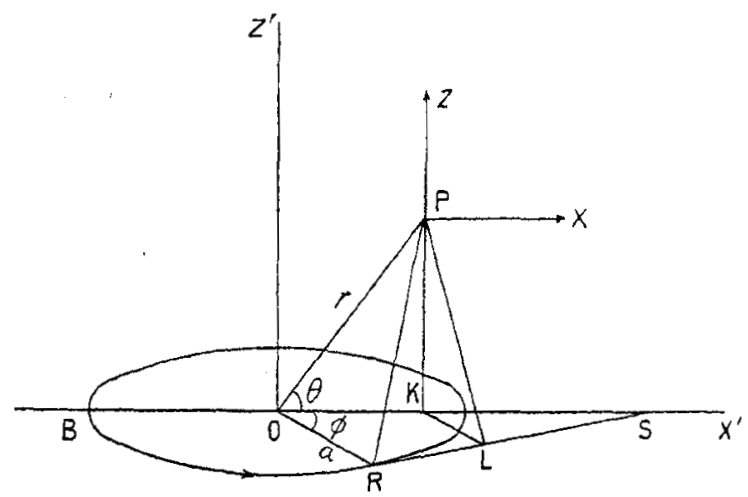

$\mathbf{X}$ and $\mathrm{Z}$ are the components of the magnetic force at $\mathbf{P}$ due to the current in the circulax filament.

that $\cos \mathrm{POR}=\cos \theta \cos \phi$, so that $\mathrm{PR}^{2}=a^{2}+r^{2}-2 a r \cos \theta \cos \phi$, we get by Laplace's formula,

$$
\begin{aligned}
d Z & =\frac{i a d \phi}{\mathrm{PR}^{2}} \sin \mathrm{PRL} \cdot \sin \mathrm{KPL} \\
& =\frac{i a d \phi}{\overline{\mathrm{PR}}^{2}} \cdot \frac{\mathrm{PL}}{\mathrm{PR}} \cdot \overline{\mathrm{PL}} \\
& =\frac{i a(a-r \cos \theta \cos \phi) d \phi}{\left(a^{2}+r^{2}-2 a r \cos \theta \cos \phi\right)^{3 / 2}} ;
\end{aligned}
$$

and therefore,

$$
\mathrm{Z}=2 i a \int_{0}^{\pi} \frac{(a-r \cos \theta \cos \phi) d \phi}{\left(a^{2}+r^{2}-2 a r^{2} \cos \theta \cos \phi\right)^{3}} .
$$


Similarly we find that

$$
\mathrm{X}=2 i a r \int_{0}^{\pi} \frac{\sin \theta \cos \phi d \phi}{\left(a^{2}+r^{2}-2 a r \cos \theta \cos \phi\right)^{3,2}} .
$$

Now (10) can be written

$$
\begin{aligned}
Z & =i \int_{0}^{\pi}\left(a^{2}+r^{2}-2 a r \cos \theta \cos \phi\right)^{1 / 2} \\
& +i\left(a^{2}-r^{2}\right) \int_{0}^{\pi} \frac{d \phi}{\left(a^{2}+r^{2}-2 a r^{2} \cos \theta \cos \phi\right)^{3 / 2}} .
\end{aligned}
$$

Hence, writing $\phi=\pi-2 \phi^{\prime}$, we get by (6) after a little reduction

Similarly

$$
\left.\begin{array}{l}
\mathrm{Z}=\frac{2 i}{r_{1}}\left(\frac{a^{2}-r^{2}}{r_{2}^{2}} \mathrm{E}+\mathrm{F}\right) \cdot \\
\mathrm{X}=\frac{2 i \tan \theta}{r_{1}}\left(\frac{a^{2}+r^{2}}{r_{2}^{2}} \mathrm{E}-\mathrm{H}\right)
\end{array}\right\},
$$

where

$$
\begin{aligned}
& r_{1}^{2}=a^{2}+r^{2}+2 a r \cos \theta, \\
& r_{2}^{2}=a^{2}+r^{2}-2 a r \cos \theta,
\end{aligned}
$$

and the modulus $h$ of the elliptic functions is given by $k^{2}=1-r_{2}^{2} / r_{1}{ }^{2}$.

If $\mathrm{R}$ and $\mathrm{T}$ be the component magnetic forces along and perpendicular to $O P$ (fig. 1), we bave

$$
\left.\begin{array}{l}
\mathrm{R}=\mathrm{Z} \sin \theta+\mathrm{X} \cos \theta=\frac{4 i a^{2} \sin \theta}{r_{1} r_{2}{ }^{2}} \mathrm{E}, . . . . . \\
\text { and } \\
\mathrm{T}=\mathrm{Z} \cos \theta-\mathrm{X} \sin \theta=\frac{2 i}{r_{1} r_{2}{ }^{2}}\left[\frac{a^{2} \cos 2 \theta-r^{2}}{\cos \theta} \mathrm{E}+\frac{r_{2}^{2}}{\cos \theta} \mathrm{F}\right]
\end{array}\right\} .
$$

In rectangular coordinates, we have

$$
\left.\begin{array}{l}
\mathrm{X}=\frac{2 i}{r_{1}}\left[\frac{2 a z}{r_{2}^{2}} \mathrm{E}-\frac{z}{x}(\mathrm{~F}-\mathrm{E})\right], \cdot \\
\mathrm{Z}=\frac{2 i}{r_{1}}\left[\frac{2 a\left(a-x^{2}\right)}{r_{2}^{2}} \mathrm{E}+(\mathrm{F}-\mathrm{E})\right],
\end{array}\right\} .
$$

where $r_{1}^{2}=(a+a)^{2}+z^{2}$,

$$
r_{2}^{2}=(a-v)^{2}+z^{2} \text {, }
$$

and

$$
k^{2}=1-r_{2}^{2} / r_{1}^{2} \text {. }
$$


If $\phi$ be the angle $\mathrm{APB}$ (fig. 2 ), $\mathrm{OP}=r$, the angle $\mathrm{POA}=\theta$, and $\mathrm{OA}=\mathrm{OB}=a$, we have $\mathrm{AP}=r_{2}$ and $\mathrm{PB}=r_{1}$.

Fig. 2.

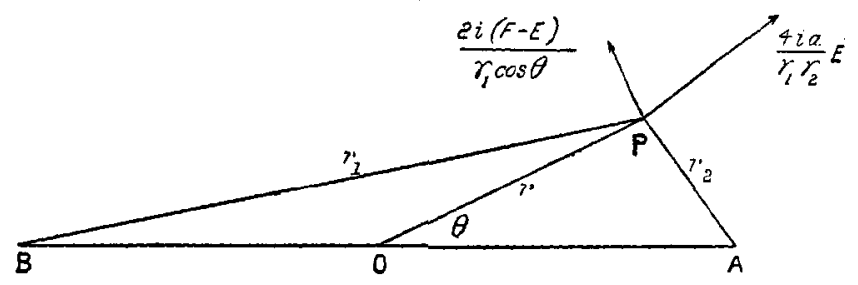

The magnetic force at $P$ due to the circular current is the resultant of the forces $2 i(\mathrm{~F}-\mathrm{E}) /\left(r_{2} \cos \theta\right)$ und $4 i a \mathrm{E} / r_{1} r_{2}$ acting at riglit angles to $\mathrm{OP}$ and $\mathrm{AP}$ respectively.

Hence in bipolar coordinates

$$
\left.\begin{array}{l}
\mathrm{Z}=-\frac{2 i}{r_{2}} \mathrm{E} \cos \phi+\frac{2 i}{r_{1}} \mathrm{~F}, . . . . . . \\
\mathrm{X}=\frac{2 i}{r_{2}} \cdot \frac{r_{1}^{2}+r_{2}^{2}}{r_{1}^{2}-r_{2}^{2}} . \mathrm{E} \sin \phi-\frac{2 i}{r_{1}} \cdot \frac{2 r_{1} r_{2}}{r_{1}^{2}-r_{2}^{2}} . \mathrm{F} \sin \phi,
\end{array}\right\} .
$$

where $\cos \phi=\left(r_{j}^{2}+r_{2}^{2}-4 a^{2}\right) /\left(2 r_{1} r_{2}\right)$, and $h^{2}=1-r_{2}^{2} / r_{1}^{2}$.

A simple and useful way of eonsidering the force at any point $\mathrm{P}$ due to a circular current is to consider that it is the resultant (fig. 2) of two forces at right angles to $A P$ and $O P$ respectively. The component at right angles to $\mathrm{AP}$ equals $\left(4 i a / r_{1} r_{2}\right) \mathrm{E}$, and the component at right angles to $O P$ equals $2 i(F-E) /\left(r_{1} \cos \theta\right)$, the modulus of $\mathrm{E}$ and $F$ being $\left(1-r_{2}^{2} / r_{1}^{2}\right)^{1 / 2}$.

4. Applications of the Formule.

i. Force in the plane of a circular current.

From fig. 2 we see at once that the force at any point in the plane of the eircular current and inside the circle is given by

$$
\begin{aligned}
\mathrm{Z} & =\frac{2 i}{r_{2}} \mathrm{E}+\frac{2 i}{r_{1}} \mathrm{~F} \\
& =\frac{2 i}{a-r} \mathrm{E}+\frac{2 i}{a+r^{2}} \mathrm{~F}, . . . .
\end{aligned}
$$

the modulus being $2(a r)^{1 / 2} /(a+r)$.

Again, at points in the plane of the circular current, but 
outside the circle, we have

$$
\begin{aligned}
\mathrm{Z} & =-\frac{2 i}{r-a} \mathrm{E}+\frac{2 i}{r+a} \mathrm{~F} \\
& =\frac{2 i}{a-r} \mathrm{E}+\frac{2 i}{a+r} \mathrm{~F} .
\end{aligned}
$$

Hence, whether the point be inside or outside the circle, formula (16) gives the magnetic force. When the point lies within the circle the author at first used the formula *

$$
\begin{aligned}
\mathrm{Z} & =\frac{4 a i}{a^{2}-r^{2}} \mathrm{E}^{\prime} \\
& =\frac{2 i}{a-r^{2}} \mathrm{E}^{\prime}+\frac{2 i}{a+r^{2}} \mathrm{E}^{\prime},
\end{aligned}
$$

where the modulus is $r / a$. By means of (9) we see that the formulæ give identical results. For points outside the circle, howerer, we find by (16) and (9), or otherwise,

$$
\mathrm{Z}=-\frac{4 r i}{r^{2}-a^{2}} \mathrm{E}^{\prime \prime}+\frac{4 i}{r^{2}} \mathrm{~F}^{\prime \prime},
$$

the modulus now being $a / r$. It will be seen that it is best to consider (16) as the standard formula, as it gives the force at points in the plane of the filament, both outside and inside the circle.

It is worth noticing that if $r$ be not greater than $0 \cdot 7 a$, the maximum inaccuracy of the formula

$$
\mathrm{Z}=\frac{\pi i}{2\left(a^{2}-r^{2}\right)}\left\{a+\sqrt{a^{2}-r^{2}}+\sqrt{4 a^{2}-2 r^{2}}\right\}
$$

is less than 1 in 10,000 .

Let us now find the value of the magnetic force very close to the filament. In this case, as the modulus of $\mathrm{E}$ and $\mathrm{F}$ in (16) is nearly unity, we can use Legendre's formulæ (1) and (2) to find $\mathrm{E}$ and $\mathrm{F}$. Hence

$$
\begin{aligned}
& \mathrm{Z}=\frac{2 i}{a-r}\left[1+\frac{1}{2}\left(\frac{a-r}{a+r}\right)^{2}\left\{\log \frac{4(a+r)}{a-r}-\frac{1}{2}\right\}+\ldots\right] \\
& +\frac{2 i}{a+r}\left[\log \frac{4(a+r)}{a-r}+\frac{1}{4}\left(\frac{a-r}{a+r}\right)^{2}\left\{\log \frac{4(a+r)}{a-r}-1\right\}+\ldots\right] \\
& =\frac{2 i}{a-r}+\frac{2 i}{a+r} \log \frac{4(a+r)}{a-r}, \ldots . . . . . .
\end{aligned}
$$

* 'The Electrician,' vol. xxxi. p. 212 (1893) or 'Alternating Currents,' vol. i. p. 30 . 
when $(a-r)^{2} /(a+r)^{2}$ is negligibly small compared with unity. The first term on the right-hand side of this equation is very great compared with the second when $r$ is nearly equal to $a$, and, in this case, we may write

$$
\mathrm{Z}=\frac{2 i}{a-r} \text {, very approximately. }
$$

Similarly, when $r$ is greater than $a$, we have

$$
\mathrm{Z}=-\frac{2 i}{r-a}+\frac{2 i}{r+a} \log \frac{4(r+a)}{r-a}, . .
$$

approximately.

\section{ii. The mutual inductance of two coaxial circular currents.}

Let the radii of the circles be $a$ and $b$ respectively, and let $c$ be the distance between their planes. We shall calculate the flux $M$ through the circle whose radius is $b$ due to unit current in the circle whose radius is $a$. Let $\mathrm{Z}$ be the magnetic force at an element $r^{\prime} d \theta^{\prime} d r^{\prime}$ of the aren of the circle whose radius is 7 , then

$$
\mathrm{M}=\int_{0}^{b} \int_{0}^{2 \pi} Z r^{\prime} d \theta^{\prime} d r^{\prime}
$$

and noticing that $r^{2}=r^{\prime 2}+c^{2}$, and $r^{2} \cos \theta=r^{\prime}$, we get

$$
\frac{M}{4 \pi a}=\int_{0}^{b} \int_{0}^{\pi} \frac{\left(a-r^{\prime} \cos \phi\right) r^{\prime} d \theta^{\prime} d r^{\prime}}{\left(a^{2}+r^{\prime 2}+c^{2}-2 a r^{\prime} \cos \phi\right)^{3 / 2}} .
$$

Thanging to rectangular coordinates, we have

$$
\begin{aligned}
\frac{\mathrm{M}}{4 \pi a} & =\int_{0}^{b} \int_{-\sqrt{b}^{2}-y^{2}}^{+\sqrt{b^{2}-y^{2}}} \frac{(a-x) d x d y}{\left\{(a-c)^{2}+y^{2}+c^{2}\right\}^{3 / 2}} \\
& =\int_{0}^{b}\left[1 /\left\{(a-x)^{2}+y^{2}+c^{2}\right\}^{1 / 2}\right]_{-\sqrt{b^{2}-y^{2}}}^{+\sqrt{b^{2}-y^{2}}} d y .
\end{aligned}
$$

Put $y=b \sin \phi$ and simplify. In the second integral also, write $\phi^{\prime}=\pi-\phi$. We get

$$
\frac{\mathrm{M}}{4 \pi a b}=\int_{0}^{\pi} \frac{\cos \phi d \phi}{\left(a^{2}+l^{2}+c^{2}-2 a b \cos \phi\right)^{1 / 2}} .
$$

Now putting $\phi=\pi-2 \theta$, we have

$$
\frac{M}{4 \pi a b}=-\frac{2}{r_{l}} \int_{0}^{\pi / 2} \frac{\cos 2 \theta}{\Delta} d \theta, \quad . \quad .
$$


and therefore, by (4)

where

$$
\mathrm{M}=4 \pi \sqrt{a b}\{(2 / k-k) \mathrm{F}-(2 / k) \mathrm{E}\}, \quad .
$$

Formula (20) agrees with that given by Helmholtz* for the analogous problem of the vortex ring.

If we transtorm (20) by the Landen-Isegendre formulæ (9), we get

where

$$
\mathrm{I}=\frac{8 \pi \sqrt{a l}}{\sqrt{k^{\prime}}}\left(\mathrm{F}^{\prime}-\mathrm{E}^{\prime}\right), \ldots . .
$$

$k^{\prime}=\left(r_{1}-r_{2}\right) /\left(r_{1}+r_{2}\right), r_{1}^{2}=(a+b)^{2}+c^{2}$, and $r_{2}^{2}=(a-b)^{2}+c^{2}$. This is Maxwell's formula.

iii. Approximate formula for the self-inductance of a ring.

We shall suppose that the radius of the ring is $a$, that the radius of the cross section is $r$, and that the current density at all points of the cross section is the same. We suppose also that $r / a$ is small compared with unity. From the construction given in fig. 2 we see that the lines of force near a circular filament are very approximately circles with their centres on the circular axis of the filament. The field is therefore similar to that round a straight cylindrical conducting tube, in which the current flow is parallel to the axis. Now it is well known $\dagger$ that the magnetic force outside a straight cylindrical conducting tube can be calculated as if the current were concentrated along the axis and that the magnetic force inside the tube is zero. Let us suppose that the circular conductor is built up of an infinite number of infinitely thin ring-tubes, every point on any tube having the same minimum distance from the circular axis, and make the assumptions that the current in each ring-tube produces no magnetic field inside it, and that the field outside can be calculated as if the current were concentrated along the axis. From the corresponding electrostatic problem of a charged conducting ring we see, by the method of duality, that the first assumption could be made rigorously true by assuming that

* Crelle's Jounnal, vol. 17. p. 25 (1858), or Ges. Abh. t. 1, p. 101.

$\dagger$ A. Russell, 'Alternatingr Currents,' vol. i, p. 32. 
the density of the current distribution over the cross section of the infinitely thin ring-tube was proportional to the surfuce density of the electrostatic charge. In this case, however, the magnetic force at points outside the tube is not the same as if the current were concentrated along the circular axis, and therefore the gain in rigour by making the first assumption accurate is problematical. When $r$ is very small compared with $a$ the surface density is approximately constant over the ring *. In this case, therefore, when the current density is uniform over the circular tube, the magnetic force produced inside is negligible, and therefore our assumptions are legitimate in obtaining an approximate solution.

Let $\Phi_{1}$ be the flux linked with the whole current when unit current is flowing in the ring, and $\Phi_{2}$ the flux linked with part of the current only. By (21) we have

$$
\Phi_{1}=8 \pi a(\mathrm{~F}-\mathrm{E}),
$$

where the modulus is $(a-r) / a$.

By (17), the force $Z$ at a point in the plane of the circular axis and at a distance $a-\xi$ from its centre is given by

$$
\mathrm{Z}=\frac{2 i^{\prime}}{\xi}+\frac{i^{\prime}}{a} \log \frac{8 a}{\xi},
$$

approximately, where $i^{\prime}=\xi^{2} / r^{2}$, since the whole current in the ring is unity.

Hence, on our assumptions

$$
\Phi_{2}=2 \pi \int_{0}^{r}(a-\xi) \mathrm{Z}\left(\xi^{2} / r^{2}\right) d \xi .
$$

This integral can be easily found. Since, however, we are supposing that $r$ and $\xi$ are very small, we may write $\mathrm{Z}=2 i^{\prime} / \xi$ simply, and thus $\Phi_{2}=\pi a$, approximately.

If $L$, therefore, denote the self-inductance, we have

$$
\begin{aligned}
\mathrm{L} & =\Phi_{1}+\Phi_{2}, \\
& =8 \pi a(\mathrm{~F}-\mathrm{E})+\pi \alpha .
\end{aligned}
$$

Substituting for $F$ and $E$ their approximate values $(1 / 2) \log (8 a / r)$ and 1 , we get approximately.

$$
\mathrm{L}=4 \pi a\{\log (8 a / n)-1 \cdot 75\}, \quad . \quad . \quad .
$$

* F. W. Dyson ${ }_{z}$ Phil. Trans, vol, elxxxiv, A, p. 67 (1893). 
The formula given by Lord Rayleigh* and Sir W. D. Niven is

$$
\mathrm{L}=4 \pi a\{\log (8 a / r)-1 \cdot 75\}+\left(\pi r^{2} / 2 a\right)\{\log (8 a / r)+1 / 3\},
$$

and by Max Wien $\uparrow$

$$
\mathrm{L}=4 \pi a\{\log (8 a / r)-1 \cdot 75\}+\left(\pi r^{2} / 2 a\right)\{\log (8 a / r)-0.0664\} \text {. }
$$

When $r / a=1 / 100$ we should expect (22) to give $L$ with considerable accuracy. We see that in this casc, the value of $\mathrm{L}$ found by it differs from that given by either Rayleigh's or Wien's formula by less than the five-hundredth part of one per cent.

If we suppose that the conductivity of the ring is infinito so that the current flows entirely on the surface, then our method of proof shows that

$$
\mathrm{L}=\Phi_{1}=8 \pi a(\mathrm{~F}-\mathrm{E})=4 \pi a\{\log (8 a / r)-2\},
$$

approximately. When $r / a=1 / 100$, the value of $L$ given by this formula is more than five per cent. lower than that given by any of the preceding formule. It will be seen, therefore, that even with a thin wire the manner in which the current is distributed over the cross section has an appreciable effect on the inductance. It must also be noticed that when $r / a$ is small, $d \mathrm{~L} / d r(=-4 \pi a / r)$ is large, and hence $L$ varies rapidly with $r$ and a small error made in measuring $r$ may lead to a large error in the calculated value of $L$.

It is interesting to compare the formula for the selfinductance of a ring with the formula for its electrostatic capacity. Formula for the capacity $\mathrm{K}$ have been found by W. M. Hicks + and F. W. Dyson \&.

When the radius of the ring is large compared with the radius of the circular section, they both agree in giving the formula as

$$
\mathrm{K}=\pi a /\{\log (8 a / r)\} .
$$

Hence, if the resistivity of the ring be zero, we have

$$
\mathrm{KL}=(2 \pi a)^{2}\{1-2 / \log (8 a / r)\} \ldots .
$$

* 'Scientific Papers,' vol. ii. p. L5.

+ Wiedemann's Annalon, liii. \}. 934 (1804).

f Phil. Trans. vol. clxxii. p. 64:3 (1881).

\$. W. Dyson, p. 68, l. c. ante.

rot. $x x$. 
When $100 r=a$, this equation becomes

$$
\mathrm{KL}=0.7(2 \pi a)^{2} \text {, approximately. }
$$

It will be seen, therefore, that we can only use the equation $\mathrm{KL}=(2 \pi a)^{2}$ when $r / a$ is an exceedingly small fraction. The corresponding equation $\mathrm{KL}=l^{2}$, for two long parallel cylinders of infinite conductivity is strictly true (see A. Russell, 'Altermating Currents,' vol. i. p. 141).

\section{Applications to Hydrodynamics.}

Lord Kelvin has shown ("Papers on Electrostatics and Magnetism,' p. 444) that the problem of finding the velocity at any point of an incompressible fluid near a vortex flament is, from the point of view of mathematical analysis, the same as the problem of finding the magnetic force near a current, filament. The strength $m$ of the vortex corresponds to the strength $i$ of the current and the fluid velocity to the magnetic force. If $r$ be the rndius of the cross section (supposed circular) of a vortex ring and $\omega$ the angular velocity of points on the sing round the circular axis, the strength * $m$ of the vortex is generally defined to be $\pi r^{2} \omega$. In the electrical problem the magnetic force tangential to the ring is $2 i / r$, and in the hydrodynamical problem the corresponding velocity is $\omega r$. Hence to convert the formula for the magnetic force at a point into the corresponding formulæ for the fluid velocity at that point we must multiply by $\omega r^{2} / 2 i$, that is, by $m / 2 \pi i$. For instance, we see from the construction given in fig. 2 that the velocity at a point $P$ in the fluid near a vortex ring can be found as follows:-The component velocity at right angles to $\mathrm{AP}$ (fig. 2) equals $\left(2 \mathrm{ma} / \pi r_{1} r_{2}\right) \mathrm{E}$, and the component at right angles to $O P$ equals

$$
m(\mathrm{~F}-\mathrm{E}) /\left(\pi r_{1} \cos \theta\right) \text {, }
$$

the modulus of $E$ and $F$ being $\left(1-r_{2}^{2} / r_{1}^{2}\right)^{1 / 2}$. At points on the surface of the vortex ring we bave $r_{2}=r$, and we may write $r_{1}=2 a, \cos \theta=1, \mathrm{E}=1$, and $\mathrm{F}=\log (8 a / r)$. Hence points on the surface of the ring have a velocity $m / \pi r$, that is, $\omega r^{\circ}$ ahout the circular axis and a linear velocity $w$ upwards,

$* 1 \mathrm{i}$ is sometimes defined as $2 \pi r^{2} \omega$. 
where

$$
w=\frac{m}{2 \pi a}\left(\log \frac{8 a}{r}-1\right) \cdot . \cdot . \quad . \quad \cdot(a)
$$

We see, therefore, that the vortex ring moves borlily upwards with the velocity $v$. We see also at onee that the velocity of the fluid at the centre of the vortex ring is $2 \pi i / a \times m / 2 \pi i$, that is, $m / a$.

Again, if the components of the velocity of an eloment of the volume $d v$ of the fluid be $u$ and $w$, and if $\rho$ be the density of the fluid, we have

$$
\begin{aligned}
\mathrm{T}_{1} & =\frac{1}{2} \rho \Sigma\left(u u^{2}+v^{2}\right) d v=\frac{\rho m^{2}}{8 \pi^{2} i^{2}} \Sigma\left(\mathrm{X}^{2}+Z^{2}\right) d v \\
& =\frac{\rho m^{2}}{\pi i^{2}} \Sigma\left(\frac{\mathrm{R}^{2}}{8 \pi}\right) d u,
\end{aligned}
$$

where $T_{1}$ is the kinetic energy of the moving fluid and $\mathrm{R}=$ the resultant magnetic force at $d v$ in the electrical problem. But by Kelvin's formula

and thus

$$
\Sigma\left(\mathrm{R}^{2} / 8 \pi\right) d v=\mathrm{L} i^{2} / 2,
$$

$$
\mathrm{T}_{1}=\frac{\rho m^{2}}{2 \pi} \mathrm{L}
$$

where $\mathrm{Li} i^{2} / 2$ is the energy due to the linkages of the lines of furce with the whole of the electric current.

Hence, by the preceding section

$$
\begin{aligned}
\mathrm{T}_{1} & =\frac{\rho m^{2}}{2 \pi} 4 \pi u\left(\log \frac{8 a}{r}-2\right), \\
& =2 \rho m^{2} a\left(\log \frac{8 a}{r}-2\right) .
\end{aligned}
$$

Let us now suppose that the core of the vortex-ring is solid so that the angular velocity of every point in it round the circular axis is constant. This corresponds to assuming that the current density over the cross section of the ring in the electrical problem is constant. Let us also suppose that the density of the core is the same as that of the fluid. The kinetic energy $\mathrm{T}_{2}$ of the core is given by

$$
\begin{aligned}
\mathrm{T}_{2} & =\frac{1}{2} \rho \pi r^{2} 2 \pi a\left\{\left(r^{2} / 2\right) \omega^{2}+w^{2}\right\}, \\
& =\frac{1}{2} \rho m^{2} a+\text { small terms. }
\end{aligned}
$$


Hence the total linetic energy of the system $\mathrm{T}_{1}+\mathrm{T}_{2}$ equals

$$
2 \rho m^{2} a\left(\log \frac{8 a}{r}-1 \cdot 75\right) \text {. }
$$

This is the formula given by Lamb*. By means of (22) we could bave written it down at once. The formula, however, Lamb gives for the velocity of translation of a vortex ring is

$$
w=\frac{m}{2 \pi a}\left(\log \frac{8 a}{r}-\frac{1}{4}\right) . \cdot \cdot \cdot(l)+
$$

This formula is due to Lord Kelvin $\ddagger$. In Basset's 'Hydrodynamics' $\$$ both formulæ are given. The author thinks that when $r / a$ is small formula $(a)$ must be the more accurate. If this be not the case, then the electrical analogy must break down at points contionous to the filament, Chree $\|$, in his paper "Vortex Rings in a Compressible Fluid,' gives formula (a). He also points ont reasons for the slight divergences in the formulæ for the motion of vortex rings given by various physicists. In another important paper $\tau$ by the same author, the similarity of the equations of vorticity to those of electrodynamics is shown very clearly. When the compressibility of the fluid is considered, the hydrodynamical problem is mnch the more difficult.

\section{The magnetic force due to a cylindrical current sheet.}

We shall suppose that there are $n$ bands of current per unit length. We shall first find the magnetic force at a point $P$ in a plane through the base of the cylinder and at a distance $r$ from the axis. Let the length of the axis of the cylinder be $l$ and its radius $a$. Then, by (10),

$$
\mathrm{Z}=2 i a \int_{0}^{\pi} \int_{0}^{l} \frac{a-r^{2} \cos \phi}{\left(z^{2}+a^{2}+r^{2}-2 a r^{2} \cos \phi\right)^{3 / 2}} \cdot n d z \cdot d \phi,
$$

where $i$ is the current in a filament.

* 'Hydrodynamics, p. 227, 3rd edition.

+ 'Hydrodynamics,' p. 227. [Note that $k=2 m$.]

+ Phil. Mag. [4] vol, xxxiii. Supp. p. 511 (1867).

$\S$ Vol. ii., (5) p. 62, and (86) p. 87.

If Proc. Edin, Math. Soc. vol. vi. p. 59 (1887).

I Proc. Edin. Math. Soc. vol. viii. p. 43 (1889). 
Hence

$$
\begin{aligned}
\mathrm{Z} & =\frac{2 n i}{l} \int_{0}^{\pi} \frac{a(a-r \cos \phi)}{\Delta^{2}}\left[\frac{z}{\left(z^{2}+\Delta^{2}\right)^{1 / 2}}\right]_{0}^{l} d \phi \\
& =2 n i \int_{0}^{\pi} \frac{a(a-r \cos \phi)}{\Delta^{2}\left(l^{2}+\Delta^{2}\right)^{2} / 2} d \phi,
\end{aligned}
$$

where $\Delta^{2}=a^{2}+r^{2}-2 a r \cos \phi$.

The magnetic force, therefore, at a point distant $h+z$ from one end and $h-z$ from the other end of a cylindrical current sheet is given by

$$
\begin{aligned}
Z=\frac{N_{1} i}{h_{1}} \int_{a}^{\pi} \frac{a(a-r \cos \phi)}{\Delta^{2}} & {\left[\frac{h_{1}+z}{\left\{\left(h_{1}+z\right)^{2}+\Delta^{2}\right\}^{1 / 2}}\right.} \\
& \left.+\frac{h_{1}-z}{\left\{\left(h_{1}-z\right)^{2}+\Delta^{2}\right\}^{1 / 2}}\right] d \phi, .
\end{aligned}
$$

where $2 h_{1}=$ the axial length of the cylinder, and $N_{1}=2 l_{1} n$.

Now noticing that

$$
\begin{aligned}
\frac{h_{1}+z}{\left\{\left(h_{1}+z\right)^{2}+\Delta^{2}\right\}^{1 / 2}}=\frac{h_{1}+z}{d} & {\left[1+\frac{1}{2} \cdot \frac{2 a r \cos \phi}{d^{2}}\right.} \\
& \left.+\frac{1}{2 \cdot 4} \cdot\left(\frac{2 a r \cos \phi}{d^{2}}\right)^{2}+\ldots\right],
\end{aligned}
$$

whero $d^{2}=\left(h_{1}+z\right)^{2}+a^{2}+r^{2}$, and that

$$
\begin{aligned}
& \int_{0}^{\pi} \frac{a\left(a-r^{2} \cos \phi\right)}{\Delta^{2}} d \phi=\pi, \\
& \int_{0}^{\pi}(2 a r \cos \phi) \frac{a\left(a-r^{2} \cos \phi\right)}{\Delta^{2}} d \phi=\pi r^{2}, \\
& \int_{0}^{\pi}\left(2 a r^{2} \cos \phi\right)^{2} \frac{a\left(a-r^{2} \cos \phi\right)}{\Delta^{2}} d \phi=\pi r^{2}\left(r^{2}+2 a^{2}\right),
\end{aligned}
$$

wo see that,

$$
\begin{aligned}
& \mathrm{Z}=\frac{\pi \mathrm{N}_{1} i}{h_{1}}\left[\frac { h _ { 1 } + z } { d } \left\{1+\frac{1}{2} \cdot \bar{r}^{2}+\frac{1.3}{2 \cdot 4} \frac{r^{2}\left(r^{2}+2 a^{2}\right)}{d^{2}}\right.\right. \\
& +\frac{1.3 .5}{2.4 .6} \cdot \frac{r^{4}\left(r^{2}+3 a^{2}\right)}{a^{6}}+\frac{1.3 .5 .7}{2.4 .6 .8} \cdot \frac{r^{1}\left(r^{4}+4 r^{2} a^{2}+6 a^{4}\right)}{d^{3}} \\
& \left.+\frac{1 \ldots 9}{2 \ldots 10^{\circ}} \cdot \frac{r^{6}\left(r^{4}+5 r^{2} a^{2}+10 u^{4}\right)}{d^{10}}+\ldots\right\} \\
& \left.+\frac{h_{1}-z}{d^{\prime}}\left\{1+\frac{1}{2} \cdot r^{2} d^{2}+\ldots\right\}\right], . . . . . .
\end{aligned}
$$

where $l^{\prime 2}=\left(h_{1}-z\right)^{2}+a^{2}+r^{2}$. 
The terms in the bracket multiplying $\left(h_{1}-z\right) / d^{\prime}$ can be found at once from the corresponding multiplier for $\left(h_{1}+z\right) / d$, by writing $d^{\prime}$ for $d$ in the terms in the latter bracket. As a rule the series converges rapidly.

Particular cases of the formula are interesting. For instance, when $h_{1}$ is infinite we get

and when $r$ is zero,

$$
\mathrm{Z}=4 \pi\left(\frac{\mathrm{N}_{1}}{2 h_{1}}\right)
$$

$$
\mathrm{Z}=\frac{\pi \mathrm{N}_{1} i}{h_{1}}\left[\frac{h_{1}+z}{d}+\frac{h_{1}-z}{d^{\prime}}\right] .
$$

Both of these results are well known. It is not diffieult to show that the latter result is true for a belical current $i$, the radius of the helix being $a$, the axial length $2 h_{1}$, and the number of turns $\mathrm{N}_{1}$.

When $z$ is zero, that is at points on the plane bisecting the axis of the cylindrical current sheet at right angles, wo have

$$
\begin{aligned}
\mathrm{Z}=\frac{2 \pi \mathrm{N}_{1} i}{\left\{h_{1}^{2}+a^{2}+r^{2}\right\}^{1 / 2}} & {\left[1+\frac{1}{2} \cdot \frac{r^{2}}{h_{1}^{2}+a^{2}+r^{2}}+\frac{3}{8} \cdot \frac{r^{2}\left(r^{2}+2 a^{2}\right)}{\left(h_{1}^{2}+a^{2}+r^{2}\right)^{2}}\right.} \\
& \left.+\frac{5}{16} \cdot \frac{r^{4}\left(r^{2}+3 a^{2}\right)}{\left(h_{1}^{2}+a^{2}+r^{2}\right)^{3}}+\ldots\right] . \quad \text {. } \quad(28)
\end{aligned}
$$

When $r^{2}$ is small compared with $h_{1}^{2}+a^{2}+r^{2}$, the series converges with great rapidity. This result, however, is not true for a helical current.

\section{Formule for the mutual inductance between two coavial cylindrical-current sheets.}

Let us suppose that there is a cylinder of radius $b$ and axial length $2 h_{2}$, coaxial and concentric with the cylinder considered above. We shall suppose that $b$ is less than $a$ and that we have $\mathrm{N}_{2}$ filaments equally spaced on the inner cylinder so that $\left.\mathrm{N}_{2 /} / 2 h_{2}\right)$. gives the turns per unit length. We have now to calculate the linkages MI of the flux, due to unit current in the onter cylinder, with the filaments of the inner cylinder. 
If $d M$ denote the linkages due to the flux through the element of area $r d \theta d r$ from $z=h_{2}$ to $z=-h_{2}$, we have

and hence, by $(26 \%$,

$$
d \mathrm{M}=\int_{-h_{2}}^{+k_{2}} \mathrm{Zr} d \theta d r \mathrm{~N}_{2}\left(d z / 2 h_{2}\right)
$$

$$
\begin{array}{r}
d \mathrm{M}=\frac{N_{1} N_{2}}{h_{1} h_{2}} \int_{0}^{b} \int_{0}^{\pi} \frac{a r d \theta(a-r \cos \phi)}{\Delta^{2}}\left[\left\{\left(h_{1}+h_{2}\right)^{2}+\Delta^{2}\right\}\right. \\
\left.-\left\{\left(h_{1}-h_{2}\right)^{2}+\Delta^{2}\right\}^{1 / 2}\right] d \phi d r .
\end{array}
$$

Therefore

$$
\begin{array}{r}
\mathrm{I}=\frac{2 \pi \mathrm{N}_{1} \mathrm{~N}_{2}}{h_{1} h_{2}} \int_{0}^{0} \int_{0}^{\pi} \frac{\operatorname{ar}(a-r \cos \phi)}{\Delta^{2}}\left[\left\{\left(h_{1}+h_{2}\right)^{2}+\Delta^{2}\right\}^{1 / 2}\right. \\
\left.-\left\{\left(h_{1}-h_{2}\right)^{2}+\Delta^{2}\right\}^{1 / 2}\right] d \phi d r . \quad . .
\end{array}
$$

It can be proved mathematically that this formula is equivalent to the following:

$$
\begin{aligned}
M=4 \pi a b e^{2} \frac{N_{1} N_{2}}{h_{1} h_{2}} \int_{0}^{\pi / 2} \frac{\sin ^{2} \theta \cos ^{2} \theta}{1-c^{2} \sin ^{2} \theta}\left[R_{1}\left(1-k_{1}^{2} \sin ^{2} \theta\right)^{1 / 2}\right. \\
\\
\left.-R_{2}\left(1-k_{2}^{2} \sin ^{2} \theta\right)^{1 / 2}\right] d \theta, . .
\end{aligned}
$$

where $\quad c^{2}=4 a b /(a+b)^{2} ; \quad R_{1}^{2}=\left(a+b_{1}\right)^{2}+\left(h_{1}+h_{2}\right)^{2}$; $\mathrm{R}_{2}{ }^{2}=(a+b)^{2}+\left(h_{1}-h_{2}\right)^{2} ; \quad k_{1}{ }^{2}=4 a b / \mathrm{R}_{1}{ }^{2}$ and $k_{2}{ }^{2}=4 a b / R_{2}{ }^{2}$. As we give a proof of the identity of (29) and (30) in the next section, it is unnecessary to give a mathematical proof here.

It is not difficult to find a series for MI by expanding the radicals in (29) by the binomial theorem and then integrating the terms. The author has found several series formulæ for M. The following two approximate formula deduced from them are simple, and when $h_{1}{ }^{2}+h_{2}{ }^{2}+a^{2}$ is greater than $l^{2}+2 a b$ they give accurate results. The first formula given (31) is much the more accurate, especially when $h_{1}$ and $h_{2}$ are not very different.

$$
\begin{aligned}
& \mathrm{M}=\frac{2 \pi \mathrm{N}_{1} \mathrm{~N}_{2}\left(\pi l^{2}\right)}{d}\left\{\frac{2 d}{\left(d^{2}+2 h_{1} h_{2}\right)^{1 / 2}+\left(d^{2}-2 h_{1} h_{2}\right)^{1 / 2}}\right. \\
& \left.+\frac{3 a^{2} b^{2}}{8 d^{ \pm}}-\frac{5 a^{2} b^{ \pm}}{16 d^{6}}\right\}, .
\end{aligned}
$$


and

$\mathrm{M}=\frac{2 \pi \mathrm{N}_{1} \mathrm{~N}_{2}\left(\pi b^{2}\right)}{d}\left\{1+\frac{3 a^{2} b^{2}+4 h_{1}{ }^{2} h_{2}{ }^{2}}{8 d^{4}}\right\}, \cdot . \cdot$

where $d^{2}=h_{1}^{2}+h_{2}^{2}+a^{2}$.

Let us suppose, for instance, that $\mathrm{N}_{1}=300, \mathrm{~N}_{2}=200, a=5$, $l=4, h_{1}=15$, and $h_{2}=2 \cdot 5$, the lengths being measured in centimetres. Formula (31) makes $\mathrm{M}=0.0011995$ henry, and (32) makes $\mathrm{M}=0.0011992$. By the complete formula given in the next section $\mathrm{M}=0.0011999$. It will be seen that the accuracy of the approximate formulae in this case is quite satisfactory.

From considerations in connexion with the magnetic potential of a cylindrical current sheet, G. F. C. Senrle* has deduced the formula

$$
\begin{aligned}
\mathrm{M}=\frac{2 \pi^{2} \mathrm{~N}_{1} \mathrm{~N}_{2} b^{2}}{d_{1}}(1 & -\frac{a^{2}}{2 d_{1}{ }^{4}} \cdot \frac{4 h_{2}{ }^{2}-3 b^{2}}{4} \\
& \left.-\frac{a^{2}\left(4 h_{1}{ }^{2}-3 a^{2}\right)}{8 d_{1}^{8}} \cdot \frac{8 h_{2}{ }^{4}-20 h_{2}{ }^{2} b^{2}+5 b^{4}}{8}-\ldots\right),
\end{aligned}
$$

where $d_{1}{ }^{2}=h_{1}^{2}+a^{2}$. The value of $M$ found by this formula in the case considered is 0.0011999 henry.

We sball find a formula in the next section by means of which $\mathrm{M}$ can always be evaluated to any specified degree of accuracy.

8. Formula for the mutual inductance between a hetiw and a coarial cylindrical survent sheet.

We shall first find Jones's formula $†$ for the mutual inductance between a circle and a coaxial helix.

From (19) we see that the mutual inductance $M$ between two coaxial circulitr eurrents is given by

$$
\mathrm{I}=-8 \pi a b \int_{0}^{\pi / 2} \frac{\cos 2 \theta d \theta}{\left\{(a+b)^{2}+z^{2}-4 a b \sin ^{2} \theta\right\}^{1 / 2}},
$$

where $z$ is the distance between their planes, and $a$ and $b$ are their radii. Hence the mutnal inductance $d \mathrm{M}$ between a

* G. F. C. Semle and J. R. Airey, 'The Electrician,' Dec. 8th, 1905.

$\dagger$ Proc. Hoy. Soc. rol. Jxii. p. 247 (1897). 
sector of the circle whose radius is $b$ and the circle whose radius is $a$ is given by

$$
d \mathrm{M}=-4 a b d \psi \int_{0}^{\pi / 2} \frac{\cos 2 \theta d \theta}{\left\{(a+b)^{2}+2^{2}-4 a b \sin ^{2} \theta\right\}^{1 / 2}}
$$

where $d \psi$ is the angle of the sector.

Let $2 \pi p$ be the pitch of the helix, and let the coordinates of points on it be given by $x=\ell \cos \psi, y=l \sin \psi$, and $z=p \psi$.

We shall first find the mutual inductance between this helix and a coaxial circle in the plane XY (fig. 3). Let us find the linknges of the belix with the flux due to unit current in the circular filament. Consider two contiguous points $P_{1}$ and $P_{2}$ on the helix and draw $P_{1} N_{1}$ and $P_{2} N_{2}$ perpendicular to $\mathrm{OZ}$. Also draw $\mathrm{P}_{2} \mathrm{P}$ parallel to $\mathrm{OZ}$ to meet the plane passing through $\mathrm{P}_{1} \mathrm{~N}_{1}$ and perpendicular to $\mathrm{OZ}$ at $\mathrm{P}$. Join $\mathrm{PN}_{1}$ and $\mathrm{PP}_{1}$. Now every line of force duo to the circular current must lie in a plane which passes through OZ. Hence, since $P_{1} P_{2}$ are infinitely close together all the lines of force linked with the circuit $\mathrm{P}_{1} \mathrm{P}_{2} \mathrm{~N}_{2} \mathrm{~N}_{1}$ pass through the sector $\mathrm{N}_{1} \mathrm{P}_{1} \mathrm{P}$. Hence if the helix have an integral number of turns and its ends be joined by a conductor lying in a plane containing the axis of the helix, we get

$$
\mathrm{M}=-4 a b \int_{0}^{\pi_{2} / 2} \int_{\psi_{1}}^{\psi_{2}} \frac{\cos 2 \theta d \psi d \theta}{\left\{(a+b)^{2}-4 a b \sin ^{2} \theta+p^{2} \bar{\psi}^{2}\right\}^{1 / 2}},
$$

where $\psi_{1}$ and $\psi_{2}$ are the values of $\psi$ at the ends of the holix.

Hence, integrating, we get

$$
\begin{aligned}
\frac{p 2 \mathrm{M}}{4 a b}=\int_{0}^{\pi / 2} & \cos 2 \theta\left[\log \left(p \psi_{1}+\sqrt{\mu^{2} \psi_{1}^{2}+\Delta^{2}}\right)\right. \\
& \left.-\log \left(p \psi_{2}+\sqrt{p^{2} \psi_{2}^{2}}+\Delta^{2}\right)\right] d \theta,
\end{aligned}
$$

where $\Delta^{2}=(a+b)^{2}-4 a b \sin ^{2} \theta$.

Integrating by parts and noticing that $\sin 2 \theta$ vanishes at both limits, we find that

$$
\begin{aligned}
\frac{p \mathrm{MI}}{4 a b} & =4 a b \int_{0}^{\pi / 2} \frac{\sin ^{2} \theta \cos ^{2} \theta d \theta}{\left(p^{2} \psi_{1}^{2}+\Delta^{2}\right)^{1 / 2}\left(p \psi_{1}+\sqrt{\left.p^{2} \bar{\psi}_{1}^{2}+\Delta^{2}\right)}-\ldots\right.} \\
& =4 a b \int_{0}^{\pi / 2} \frac{\sin ^{2} \theta \cos ^{2} \theta}{\Delta^{2}\left(p^{2} \psi_{1}^{2}+\Delta^{2}\right)^{3 / 2}}\left(\sqrt{p^{2} \psi_{1}^{2}+\Delta^{2}-\nu^{2} \psi_{1}}\right) d \theta-\ldots \\
& =4 a l \int_{0}^{\pi / 2} \frac{\sin ^{2} \theta \cos ^{2} \theta}{\Delta^{2}}\left\{\frac{\eta_{\psi_{2}}}{\sqrt{p^{2} \psi_{2}^{2}+\Delta^{2}}}-\frac{m_{1}}{\sqrt{p^{2} \psi_{1}^{2}}+\Delta^{2}}\right\} d \theta .
\end{aligned}
$$


Tig. 3.

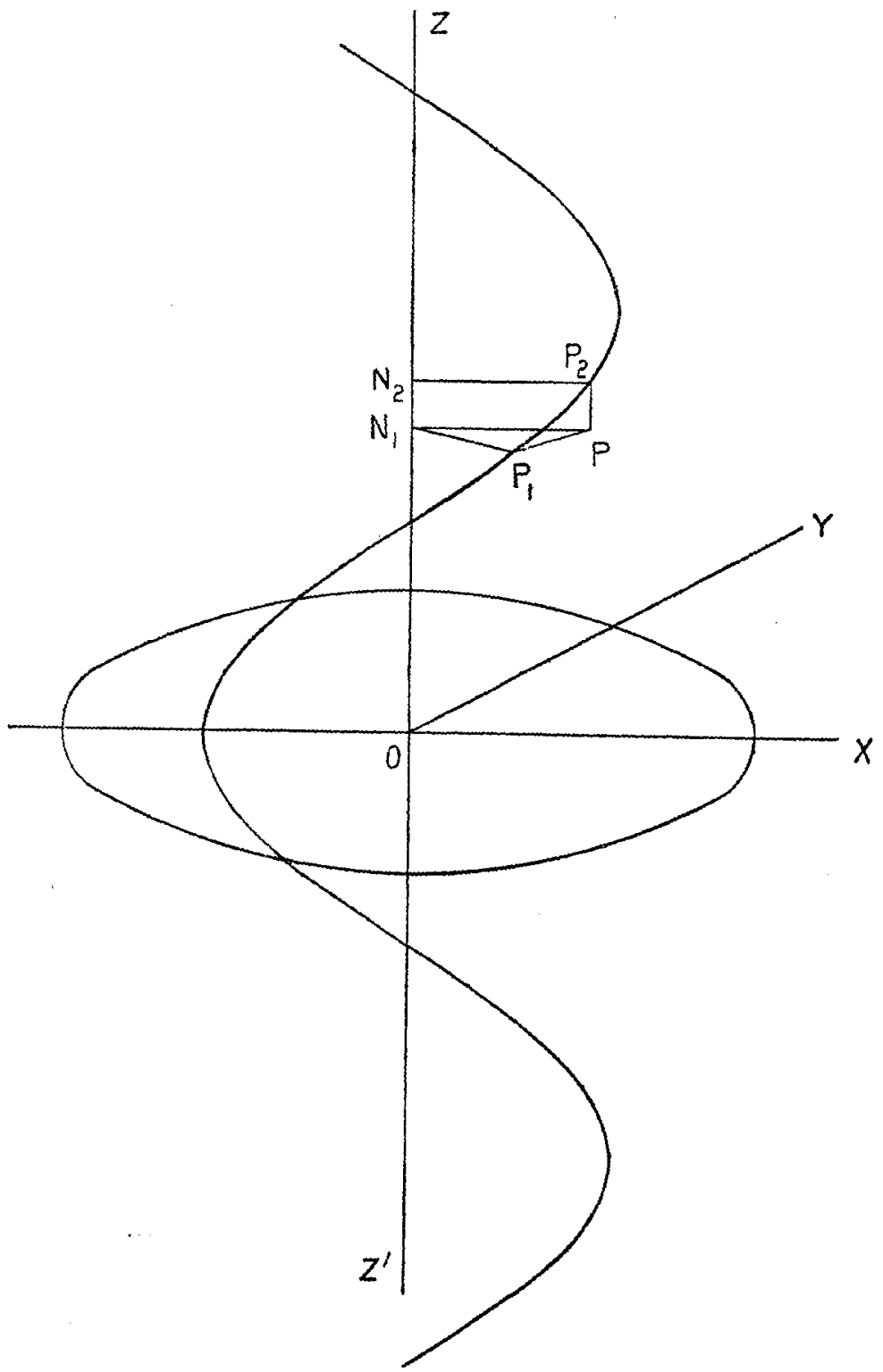

Ifelix and Circle. ZOZ' is the axis of the holix and XOY is the plane of the circle. The axinl length of the helix is $2 h_{3}$, its radius $b$ and the number of turns $\mathrm{N}_{1}$. The dadius of the circle is $a$. 
Therefore

$$
\frac{M}{4 a b}=c^{2} \int_{0}^{\pi / 2} \frac{\sin ^{2} \theta \cos ^{2} \theta}{1-c^{2} \sin ^{2} \theta}\left\{\frac{\psi_{2}}{R_{2} \Delta_{2}}-\frac{\psi_{1}}{R_{1} \Delta_{1}}\right\} d \theta,
$$

where $\quad c^{2}=4 a b /(a+b)^{2} ; \quad R_{\perp}^{2}=(a+b)^{2}+p^{2} \psi_{1}^{2}$;

$$
\begin{aligned}
& \mathrm{R}_{2}{ }^{2}=(a+b)^{2}+p^{2} \psi_{2}{ }^{2} ; \quad k_{1}{ }^{2}=4 a b / R_{1}{ }^{2} ; \quad k_{2}{ }^{2}=4 a b / R_{2}{ }^{2} ; \\
& \Delta_{1}{ }^{2}=1-k_{1}{ }^{2} \sin ^{2} \theta, \quad \text { and } \quad \Delta_{2}{ }^{2}=1-k_{2}{ }^{2} \sin ^{2} \theta .
\end{aligned}
$$

If $-\psi_{1}=\psi_{2}=\psi, R_{1}=R_{2}=R$, and $k^{2}=4 a l / R^{2}$, we havo

$$
\frac{M}{4 a b}=\frac{c^{2} \Psi}{M} \int_{0}^{\pi / 2} \frac{\sin ^{2} \theta \cos ^{2} \theta d \theta}{\left(1-c^{2} \sin ^{2} \theta\right) \sqrt{1-k^{2} \sin ^{2} \theta}} .
$$

Noticing that

$$
c^{2} \sin ^{2} \theta \cos ^{2} \theta=-\frac{1-c^{2}}{c^{2}}+\left(1-c^{2} \sin ^{2} \theta\right)\left(\sin ^{2} \theta+\frac{1-c^{2}}{c^{2}}\right),
$$

and using (3), we get

where

$$
M=2 \psi(a+b) c k\left[\frac{1}{k^{2}}(\mathrm{~F}-\mathrm{E})+\frac{1-c^{2}}{c^{2}}(\mathrm{~F}-\Pi)\right], .
$$

$$
\Pi=\int_{0}^{\pi / 2} \frac{d \theta}{\left(1-c^{2} \sin ^{2} \theta\right)\left(1-k^{2} \sin ^{2} \theta\right)^{1 / 2}},
$$

and is consequently the complete elliptic integral of the third kind in which $n=-c^{2}$. This is the final form in which Jones gave his formula.

If we pat $\psi=\pi$, and $p=0$, so that $c=k$, then, by $(6)$, wo get

$$
\mathrm{M}=4 \pi \sqrt{\overline{a b}}\{(2 / k-k) \mathrm{F}-(2 / k) \mathrm{E}\},
$$

which is formula (20).

M may be found from (35) by a formula, due to Legendre, given on p. 138 of Vol. I. of his Traité des Fonctions Elliytiques, and by the tables given in Vol. II.

Legendre's formula may be written in the form

$$
\begin{aligned}
\mathrm{F}-\Pi=\frac{c}{k^{2} \sin \alpha \cos \alpha}\left[\mathrm{E} \cdot \mathrm{F}\left(k^{\prime}, \alpha\right)+\mathrm{F} \cdot \mathrm{E}\left(k^{\prime}, \alpha\right)\right. \\
\\
\left.-\mathrm{F} \cdot \mathrm{F}\left(k^{\prime}, \alpha\right)-\pi / 2\right] . \quad(36)^{*}
\end{aligned}
$$

* Set also Cayley's 'Elliptic Functions,' Chrapter V. p. 139, 2nd edition. 
In this formula $k^{\prime 2}=1-k^{2}$, and $\mathrm{F}\left(k^{\prime}, \alpha\right), \mathrm{E}\left(k^{\prime}, \alpha\right)$ reprresent elliptic integrals of modulus $k^{\prime}$ and amplitude $\alpha$, where $\sin \alpha=\left(1-c^{2}\right)^{1 / 2} / k^{\prime}$.

We shall now find the mutual inductance between a helix and a coaxial concentric cylindrical current sheet. Let $\mathrm{N}_{1}$ be the number of turns of the helix, $2 h_{1}$ its axial length, and $a$ its radius. If $p$ be the pitch of the helix, $h_{1}=p \mathrm{~N}_{1} \pi$. Let also $\mathrm{N}_{2}, 2 h_{2}$, and $b$ be the number of turns, axial length and radius of the cylindrical sheet. Let us consider a filament of the cylinder at a height $z$ above the median plane. Then its distances from the onds of the axis of the helix will bo $h_{1}+z$ and ${ }_{1}-z$ respectively. Hence if $d \mathrm{M}$ be the linkages of the flax due to unit current in the helix embraced by the circular filament $\mathrm{N}_{2}\left(d z / 2 h_{2}\right)$, we get by (33)

$$
\begin{aligned}
d \mathrm{M}=\frac{4 a l, c^{2}}{p} \int_{0}^{\pi / 2} \frac{\sin ^{2} \theta \cos ^{2} \theta}{1-c^{2} \sin ^{2} \theta} & {\left[\frac{h_{1}+z}{\left\{\left(h_{1}+z\right)^{2}+\Delta^{2}\right\}^{1 / 2}}\right.} \\
& \left.+\frac{h_{1}-z}{\left\{\left(h_{1}-z\right)^{2}+\Delta^{2}\right\}^{1 / 2}}\right] d \theta \frac{\mathrm{N}_{2} d z}{2 h_{2}},
\end{aligned}
$$

and thercfore, integrating from $-h_{2}$ to $+h_{2}$ we get

$$
\begin{array}{r}
M=4 \pi a b c^{2} N_{1} N_{2} \int_{1} h_{2} \int_{0}^{\pi / 2} \sin ^{2} \theta \cos ^{2} \theta \\
1-c^{2} \sin ^{2} \theta \\
- \\
\left.-R_{2}\left(1-k_{2}{ }^{2} \sin ^{2} \theta\right)^{1 / 2}\right] d \theta .
\end{array}
$$

Now

$$
\begin{aligned}
& c^{2} \sin ^{2} \theta \cos ^{2} \theta \sqrt{1-k_{1}^{2} \sin ^{2} \theta} \\
& =\frac{\left(c^{2}-k_{1}^{2}\right) \sin ^{2} \theta \cos ^{2} \theta+k_{1}^{2} \sin ^{2} \theta \cos ^{2} \theta\left(1-c^{2} \sin ^{2} \theta\right)}{\sqrt{1-k_{1}^{2} \sin ^{2} \theta}},
\end{aligned}
$$

and thus, by (5)

$$
\begin{aligned}
& \mathrm{H}= \pm \pi c l \frac{\mathrm{N}_{1} \mathrm{~N}_{2}}{h_{1} h_{2}}\left[R_{1}\left(1-\frac{k_{1}^{2}}{c^{2}}\right)\left\{\frac{1-c^{2}}{c^{2}}\left(F_{1}-I_{1}\right)+\frac{1}{k_{1}^{2}}\left(\mathrm{~F}_{1}-\mathrm{E}_{1}\right)\right\}\right. \\
& +\mathrm{R}_{1} k_{1}^{2}\left\{\frac{2-k_{1}^{2}}{3 k_{1}^{2}} \mathrm{E}_{1}-\frac{2-2 k_{1}^{2}}{3 k_{1}^{2}} \mathrm{~F}_{1}\right\} \\
& \left.-R_{2}\left(1-\frac{k_{2}{ }^{2}}{c^{2}}\right)\left\{\frac{1-c^{2}}{c^{2}}\left(\mathrm{~F}_{2}-\Pi_{2}\right)+\frac{1}{l_{2}^{2}} \mathrm{~F}_{2}-\mathrm{E}_{2}\right)\right\}
\end{aligned}
$$

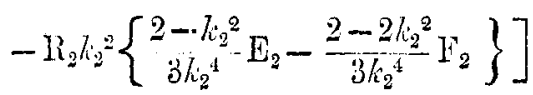




$$
\begin{aligned}
=4 \pi a 7) \frac{N_{1} N_{2}}{l_{1} h_{2}}[ & R_{1}\left(1-\frac{k_{1}^{2}}{c^{2}}\right)\left(\frac{1-c^{2}}{c^{2}}\right)\left(\mathrm{F}_{1}-\mathrm{H}_{1}\right) \\
& +R_{1}\left(\frac{1}{3 k_{1}^{2}}-\frac{1}{c^{2}}+\frac{1}{3}\right)\left(\mathrm{F}_{1}-\mathrm{E}_{1}\right)+\frac{1}{3} R_{1} \mathrm{~F}_{1} \\
& -R_{2}\left(1-\frac{k_{2}^{2}}{c^{2}}\right)\left(\frac{1-c^{2}}{c^{2}}\right)\left(\mathrm{F}_{2}-\Pi_{2}\right) \\
& \left.-R_{2}\left(\frac{1}{3 k_{2}^{2}}-\frac{1}{c^{2}}+\frac{1}{3}\right)\left(\mathrm{F}_{2}-\mathrm{E}_{2}\right)-\frac{1}{3} R_{2} \mathrm{~F}_{2}\right] .
\end{aligned}
$$

By the aid of (36), therefore, and the tables given in Legendre's treatise, M may be calculated by this formula to any desired accuracy. If a four-figure accuracy snffice, the tables given on pp. 68-75 of Dale's 'Mathematical Tables' will be found imple.

It is to be noticed that provided $a, b, h_{1}$ and $h_{2}$ remain the same, the formula for the mutual inductance between a helical current and a cylindrical current sheet may be written in the form $m \mathrm{~N}_{1} \mathrm{~N}_{2}$ where $m$ is a constant. By considering the case, therefore, of $N_{1}$ infinitely great, that is, when the helix is practically identical with a cylindrical current sheet of the same axial length and diameter, we see that the formula for two cylindrical current sheets must also be $m \mathrm{~N}_{1} \mathrm{~N}_{2}$. We thus see that the formula (29) and (38) must be identical.

It las to be remembered that the above formula needs to be modified when both the coils are helical. If the pitch of one of the helices be a very small quantity the correcting factor will be small. It is advisable, however, when actually measuring the mutual inductance to investigate whether rotating * one of the helices round the axis through varions angles alters the mutual inductance.

Eren when Legendre's tables are accessible, the evaluation of $(38)$ is laborious. We shall, therefore, give a series formula for $M$ which is convenient in many cases. By the binomial theorem, we always have

$$
\begin{aligned}
& \int_{0}^{\pi / 2} \frac{\sin ^{2} \theta \cos ^{2} \theta}{1-c^{2} \sin ^{2} \theta}\left(1-k_{1}^{2} \sin ^{2} \theta\right)^{1,2} d \theta \\
& =\mathrm{P}_{1}-\frac{1}{2} \mathrm{P}_{2} h_{1}{ }^{2}-\frac{1.1}{2.4} \mathrm{P}_{3} k_{1}-\frac{1.1 .3}{2.4 .6} \mathrm{P}_{4} h_{1}{ }^{6}-\ldots \text {, }
\end{aligned}
$$


500

whore

$$
\begin{aligned}
P_{n} & =\int_{0}^{\pi / 2} \frac{\sin ^{2 n} \theta \cos ^{2} \theta}{1-c^{2} \sin ^{2} \theta} d \theta \\
& =\frac{P_{n-1}}{c^{2}}-\frac{1}{c^{2}} \int_{0}^{\pi / 2} \sin ^{2 n-2} \theta \cos ^{2} \theta d \theta \\
& =\frac{P_{n-1}}{c^{2}}-\frac{1}{2 n} \cdot \frac{1.3 .5 \ldots 2 n-3}{2,4.6 \ldots 2 n-2} \cdot \frac{\pi}{2} \cdot \frac{1}{c^{2}} . . .
\end{aligned}
$$

Now it is easy to show that

$$
\mathrm{P}_{1}=\frac{\pi}{4\left(1+\sqrt{\left.1-c^{2}\right)^{2}}\right.}=\frac{\pi}{16} \cdot \frac{(a+b)^{2}}{a^{2}},
$$

and if we denote $P_{n} / P_{1}$ by $\eta_{n}$ we get

$$
q_{n}=\frac{(a+b)^{2}}{4 a b} q_{n-1}-\frac{1}{n} \cdot \frac{1 \cdot 3 \cdot 5 \ldots 2 n-3}{2 \cdot 4 \cdot 6 \ldots 2 n-2} \cdot \frac{\alpha}{b} .
$$

Thus the successive values of $q_{n}$ can be readily computel.

Since $P_{n}$ is always less than $P_{n-1}$, and $l_{1}$ is always less than unity, it follows that the series given above is always convergent. The formula for $M$ can, therefore, be written in the form

$$
\begin{aligned}
M=\pi^{2} b^{2} \frac{N_{1} N_{2}}{h_{1} l_{2}}\left[R_{1}\right. & \left\{1-\frac{1}{2} q_{2} k_{1}{ }^{2}-\frac{1.1}{2.4} q_{3} k_{1}{ }^{4}-\ldots\right\} \\
- & \left.R_{2}\left\{1-\frac{1}{2} q_{2} k_{2}{ }^{2}-\frac{1.1}{2.4} q_{3} k_{2}{ }^{4}-\ldots\right\}\right],
\end{aligned}
$$

where $R_{1}^{2}=(a+b)^{2}+\left(h_{1}+h_{2}\right)^{2} ; l_{1}^{2}=4 a b / R_{1}^{2}$, and $\mathrm{R}_{2}{ }^{2}=(a+b)^{2}+\left(h_{1}-h_{2}\right)^{2} ; k_{2}{ }^{2}=4 a b / \mathrm{R}_{2}{ }^{2}$.

This formula gives a complete practical solution of the problem.

Let us take the case of the two cylindrical coils for which $a=5, l=4, \vec{h}_{1}=15, h_{2}=2 \cdot 5, \mathrm{~N}_{1}=300$, and $\mathrm{N}_{2}=200$, mentioned in the preceding section. By (39) we easily oltain

$$
\begin{aligned}
& q_{2}=0.7, \quad q_{3}=0.5525, \quad q_{1}=0.4618, \quad \eta_{1}=0.3992, \\
& q_{0}=0.3527, \quad q_{7}=0.3170, \quad \text { \&c. }
\end{aligned}
$$

* Lord Rayleigh, British Associntion Report (1899), p. 241. 
Also $\quad R_{1}^{2}=(a+b)^{2}+\left(h_{1}+h_{2}\right)^{2}=387.25 ; \quad R_{1}=19.6787 ;$

$$
\begin{aligned}
& R_{2}{ }^{2}=(a+l)^{2}+\left(h_{1}-h_{2}\right)^{2}=237 \cdot 25 ; \quad R_{2}=15 \cdot 4029 ; \\
& k_{1}{ }^{2}=80 / 387 \cdot 25 ; \quad k_{2}{ }^{2}=80 / 237 \cdot 25 .
\end{aligned}
$$

Thus, substituting in (41), we have

$$
\begin{aligned}
& M=\frac{(4 \pi)^{2} 6.10^{4}}{37 \cdot 5}[19.6787(1-0.07230-0.00295 \\
&-0.00026-0.00003-\ldots) \\
&-15.4029(1-0.11802-0.00785 \\
&-0.00111-0.00020 \\
&-0.00004-0.00001-\ldots)] 10^{-0} \\
&=0.0011999 \text { henry }, \quad
\end{aligned}
$$

which agrees with the answer found from Scarle's formula.

When $h_{1}=h_{2}=h$,

$$
k_{1}^{2}=4 a b /\left\{(a+b)^{2}+4 l^{2}\right\} \text { and } k_{2}^{2}=4 a l /(a+b)^{2}=c^{2} \text {. }
$$

In this case the series of terms with the suffix 2 in (41) may converge slowly. It is better, therefore, to express the seconi? half of the formula on the right-hand side of $(41)$ in terms of elliptic integrals. The formula * now becomes:

$$
\begin{aligned}
M=\pi^{2} b^{2} \frac{\mathrm{N}_{1} \mathrm{~N}_{2}}{h^{2}}\left[R_{1}\left\{1-\frac{1}{2} q_{2} k_{1}{ }^{2}-\frac{1.1}{2.4} q_{3} k_{1}^{4}-\frac{1.1 \cdot 3}{2.4 .6} q_{1} k_{1}^{6}-\ldots\right\}\right] \\
+\frac{8 \pi a b)}{3(a+b)} \cdot \frac{\mathrm{N}_{1} \mathrm{~N}_{2}}{h_{l^{2}}}\left[\left(a^{2}+b^{2}\right)(\mathrm{F}-\mathrm{E})-2 a b \mathrm{~F}\right],
\end{aligned}
$$

where the modulus of the elliptic functions is $2(a b)^{1 / 2} /(a+b)$.

For the calculation of the mutual inductance standards required for calibrating the galvanometers used in irontesting, this formula is suitable. In order that the coils be as compact as possible, it is necessary to malie $h=a$ and $b$ large, but even in this case the series can be ensily summed.

Let us now consider the case when the cylinder has the same radius as the helix. We may suppose, for instance, that both coils are wound on the same insulating cylinder. The

* For auother formula in terms of incomplete elliptic integrals, see J. G. Coffin, p. 124, l. c. ante. 
formula (37) now becomes

$$
\begin{aligned}
M=4 \pi \alpha^{2} \frac{N_{1} N_{2}}{h_{1} h_{2}} \int_{0}^{\pi \cdot 2} \sin ^{2} \theta & {\left[R_{1}\left(1-h_{1}^{2} \sin ^{2} \theta\right)^{1 / 2}\right.} \\
& \left.-R_{2}\left(1-h_{2}^{2} \sin ^{2} \theta\right)^{1 / 2}\right] d \theta .
\end{aligned}
$$

Hence, from (8),

whero

$$
\begin{aligned}
M=4 \pi a^{2} \frac{N_{1} N_{2}}{h_{1} h_{2}}\left[R_{1} \frac{2 k_{1}^{2}-1}{3 k_{1}^{2}} \mathrm{E}_{1}+R_{1} \frac{1-k_{1}^{2}}{3 k_{1}^{2}-F_{1}}\right. & \\
& \left.\quad-R_{2} \frac{2 k_{2}^{2}-1}{3 k_{2}^{2}} \mathrm{E}_{2}-R_{2} \frac{1-l_{2}^{2}}{3 k_{2}^{2}} \mathrm{~F}_{y}\right],
\end{aligned}
$$

$$
\begin{gathered}
R_{1}^{2}=4 a^{2}+\left(h_{1}+h_{2}\right)^{2}, \quad R_{2}{ }^{2}=4 a^{2}+\left(h_{1}-h_{2}\right)^{2}, \\
k_{1}^{2}=4 a^{2} / R_{1}^{2}, \text { and } k_{2}^{2}=4 a^{2} / R_{2}^{2} .
\end{gathered}
$$

The value of $\mathrm{M}$ can be readily found from this formula with the help of Dale's Mathematical Tables or by the series for $\mathrm{E}$ and $\mathrm{F}$ given in $\S 2$.

If we have, in addition, $h_{1}=h_{2}=h$, then

$$
R_{1}=2\left(a^{2}+k^{2}\right)^{1,2}, R_{2}=2 a, k_{1}^{2}=a^{2} /\left(a^{2}+l^{2}\right) \text {, and } k_{2}^{2}=1 \text {. }
$$

Hence

$$
\mathrm{M}=\frac{8 \pi \iota^{2}}{3 h} \mathrm{~N}_{1} \mathrm{~N}_{2}\left[\left(1+\frac{a^{2}}{h^{2}}\right)^{1 / 2}\left\{\mathrm{E}_{1}+\frac{h^{2}}{u^{2}}\left(\mathrm{~F}_{1}-\mathrm{E}_{1}\right)\right\}-\frac{a}{h}\right] .
$$

If the height $2 h$ equal the diameter $2 a$, so that the coils are as compact as possible, we may write

$$
\mathrm{M}=13.589 \mathrm{~N}_{1} \mathrm{~N}_{2} l \text {. . . . . . }
$$

9. Formula for the self-inductance of a helical current when the pitch of the helia is small.

If we have $a$ wire closely wound on a smooth insulating cylinder, the diameter of the wire being small compared with that of the cylinder, then the mutual inductanee between it and a cylindrical current sheet of the same radius as the helix formed by the axis of the wire and having the same axial length, will be approximately equal to the self- 
inductance $\mathrm{L}$ of the coil. Hence, by (44), we find that

$$
\mathrm{L}=\frac{8 \pi a^{2}}{3 h} \mathrm{~N}^{2}\left[\left(1+\frac{a^{2}}{h^{2}}\right)^{1 / 2}\left\{\mathrm{E}_{1}+\frac{h^{2}}{a^{2}}\left(\mathrm{~F}_{1}-\mathrm{E}_{1}\right)\right\}-\frac{a}{h}\right]
$$

where $\mathrm{N}$ is the number of turns of the helix and $a /\left(a^{2}+l^{2}\right)^{1,2}$ is the modulus of the elliptic functions.

From a paper by Rosa *, the author has recently discovered that this formula has been previously given by Lorenz $\dagger$. Before finding this out, however, he had given the formula to his students, and several of them had constructed standards of self-inductance by simply turning cylinders of well-seasoned teak in a lathe and winding cotton-covered wire round them. The agreement between measurement and calculation was found quite satisfactory.

When $h$ is less than $a$, we get by the Binomial Theorem and (1) and (2),

$$
\mathrm{L}=4 \pi a \mathrm{~N}^{2}\left[\log \frac{4 a}{h}-\frac{1}{2}+\frac{h^{2}}{8 a^{2}}\left(\log \frac{4 a}{h}+\frac{1}{4}\right)\right] .
$$

This is in exact agreement with a formula previously given by Lord Rayleigh ('Scientific Papors,' vol. ii. p. 15).

When $h$ equals (3/4) $a$, the number found by this formulit is too small by about the quarter of one per cent. For smaller values of $h$ the numbers found by $(46)$ and $(47)$ are in practical agreement. When $h$ is small, a small percentage error in determining it introduces a large percentage error into the calculated value of $L$. Hence, in malking standards, it is advisable to have $h$ not less than $2 a$. In this case we can use the following remarkably simple formula :

$$
\mathrm{L}=\frac{2 \pi^{2} a^{2}}{h} \mathrm{~N}^{2}\left[1-\frac{4 a}{3 \pi h}+\frac{a^{2}}{8 h^{2}}-\frac{a^{4}}{64 h^{4}}\right] .
$$

When $h=2 a$, the inaccuracy of the formula (48) as compared with the more accurate elliptic integral formula is less

* Bull. Bureau of Standards, p. 162, Aug. 1906.

$\dagger$ Wied. Ann, vii. p. 161 (1879).

$\ddagger$ The much more elaborate formula given by Collin (l. e. ante, p, 11:3) may be deduced in the same way from $(46)$.

roL. XX. 
than 1 in 8000 . If $h$ be greater than $2 a$, therefore, it is sufficiently accurate for all practical purposes.

It is convenient to write

$$
\mathrm{L}=\beta\left(a^{2} / h\right) \mathrm{N}^{2}, \quad \cdot \quad \cdot \cdot \cdot
$$

and tabulate the values of $\beta$ for various values of $l / \alpha$ :-

\begin{tabular}{|c|c|c|c|c|c|c|c|c|}
\hline$h / a \ldots \ldots$. & $1 / 100$. & $1 / 10$. & $1 / 5$. & $1 / 4$. & $1 / 2$. & $3 / 4$. & 1. & 2. \\
$\beta \ldots \ldots \ldots$. & 0.6901 & 4.013 & 6.313 & $7 \cdot 214$ & 10.38 & 12.34 & 13.59 & 16.15 \\
\hline$h / a \ldots \ldots .$. & 3. & 4. & 5. & 6. & 7. & 8. & 9. & 10. \\
\hline$\beta \ldots \ldots .$. & $17 \cdot 22$ & 17.80 & 18.16 & 18.41 & 18.59 & 18.73 & 18.84 & 18.01 \\
\hline
\end{tabular}

When $h$ is greater than $40 a$ we may write

$$
\mathrm{L}=19 \cdot 74(1-4 a / 3 \pi h)\left(a^{2} / \lambda\right) \mathrm{N}^{2} ; \quad \text {. . }
$$

and when $h$ is greater than $400 a$, we get the well-known formula

$$
\mathrm{L}=19 \cdot 74\left(a^{2} / h\right) \mathrm{N}^{2}
$$

[Note added April 15, 1907.]-Kirchhoff * in 1864 found a series formula for the self-inductance of a helical current by supposing that it is exactly equivalent to a series of parallel circular currents $\dagger$, and Strasser $\ddagger$ has elaborated the formula. Rosa $\$$ has worked out numerical examples, and it is interesting to find that in the cases considered the difference between the results computed by the series and the Jurenz formula is small. It must be noticed, however, that for very thin wires wound on large cylinders the two formulæ give very different results. The anthor has not yet worked out the exact formula taking the helicity into

* Ges. Abh. p. 170 .

$\uparrow$ The strength of the magnetic field at all points on the axis of the coil is the same in the two cases but it is not the same at all other points.

\pm Ann. 1. Phys, xvis. 1. 763, I605.

$\S$ L. c. ante. 
ascount, but judging from his experimental results he considers the Lorenz formula more accurate than the series formula when the wires are thin and the diameter of the helix is large.

The following description of two cylindrical coils wound by the author's assistant, Mr. J. N. Alty, is of interest as it shows that a mutual inductance standard having a maximum inaccuracy of less than the half of one per cent. may be constructed in a few hours. Both coils are wound on ordinary glass cylinders similar to those supplied for instrument covers. The mean diameter of the outer cylinder is $20.50 \mathrm{~cm}$., and of the inner $19 \cdot 03$. The outer cylinder is wound with 174 turns of No. 20 double silk-covered copper wire, and the inner with 289 turns of No. 24 duuble sill-covered copper wire. The axial length $(2 h)$ of each coil is $19.0 \mathrm{~cm}$., and the diameters $(2 a$ and $2 \zeta$ ) of the two helical axes of the wires are 20.60 and $19.09 \mathrm{~cm}$. respectively. By (42) we find that

$$
\mathrm{M}=0.00624 \text { heury, }
$$

and this was found to agree within the half of one per cent. with the value found by comparing it with a standard condenser.

The self-inductance coefficients of the two cylindrical coils can also be readily calculated by (46). We find that

$$
L_{1}=0.00447 \text { and } L_{2}=0.0106 \text { henry. }
$$

These numbers agree within one per cent. with the measured vilues.

The leakage factor $\sigma$ of the coils is given by

$$
\sigma=1-\mathrm{N}^{2} / \mathrm{L}_{1} \mathrm{~L}_{2}=0 \cdot 177 \text {. }
$$

The author finds it useful when experimenting with Duddell currents to make the coils of his air-core transformers cylindrical in shape, as the inductunce cocfticients can then be readily calculated.

\section{Discusston.}

Mr. A. Canpoell anid the accurate calculation of mutual inductances was of great practical importance because it formed the basis of severul of the chief mothods of determining the absolute clectrical units: it was therefore highly 


\section{THE MAGNETIC FIELD OF CYLINDRIOAL CUIIEATRS.}

satisfactory that Mr. Russell had succeeded in obtiining the complete solution for the most general case required. The author's series solution (41) was a most interesting one. When the length of one of the coils was very small compared with that of the other the series became practically $\% / 0$. This was the case used by Prof. Jones. Mr. Campbell suggested that from the author's series formula a suitable series might be obtained by differentiating with regard to $h_{2}$ (omitting the $h_{2}$ in the denominator). In calculating mutual inductance by Mr. Russell's complete formula (38) or by that of Prof. Viriamu Jones, it was necessary to find the incomplete integrals $\mathrm{E}(c, \phi)$ and $\mathrm{F}(c, \phi)$. These could often be found to a high degree of accuracy by the following two formula due to Legendre, viz. :

and

$$
\mathrm{E}(c, \phi)=\frac{1}{c} \sin c \phi+\frac{l^{2} c^{2}}{30} \phi^{5}-\frac{l^{2} c^{2}}{1260} \phi^{7}\left(4-11 c^{2}\right),
$$

$$
F(c, \phi)=\frac{1}{c} \log _{e}\left(\frac{\pi}{4}+\frac{c \phi}{2}\right)-\frac{l^{2} c^{2}}{30} \phi^{5}+\frac{l^{2} c^{2}}{1260} \phi^{7}\left(4-41 c^{2}\right),
$$

where $l=1-c^{2}$. The first formula is accurate to 7 decimal places if $\phi<30^{\circ}$ and the second to a similar accuracy if $\phi<20^{\circ}$ or if $\phi<30^{\circ}$ when $c<\sin 35^{\circ}$ or $>\sin 75^{\circ}$.

Mr. A. Russeld, in reply, after thanking Mr. Campbell for his remarks, called attention to the important relations between the equations of vorticity in hydrodynamics and the equations of electrodynamics. $\mathrm{He}$ had experienced difficulty in conceiving the nature of the stress in the medium surrounding an electric current. Was the energy stored in the medium when a current was flowing linetic or potential? Some physicists spoke as if the state of stress in the medium was due to streams of ether whirling round the electric current; others, that it was due to a compression of the medium. 